\title{
Perioperative changes in cortical excitability, mood, and quality of life in patients with primary hyperparathyroidism: a pilot study using transcranial magnetic stimulation
}

\author{
A Hermsen, A Eienbröker, A Haag, V Mylius, H M Hamer ${ }^{1}$, K Menzler, \\ E Karakas ${ }^{2, *}$ and F Rosenow* \\ Department of Neurology, Epilepsy Centre Hessen, Philipps-University Marburg, Baldingerstr., 35043 Marburg, \\ Germany, ${ }^{1}$ Department of Neurology, Epilepsy Centre Erlangen, University Erlangen, Erlangen, Germany and \\ ${ }^{2}$ Department of General and Visceral Surgery, Dr.-Horst-Schmidt Hospital, Wiesbaden, Germany \\ *(E Karakas and $\mathrm{F}$ Rosenow contributed equally to the study)
}

\author{
Correspondence \\ should be addressed \\ to A Hermsen \\ Email \\ hermsen@ \\ staff.uni-marburg.de
}

\begin{abstract}
Objective: Serum calcium $\left(\mathrm{Ca}^{2+}\right)$ and parathyroid hormone (PTH), amongst others, modify cortical excitability. Alterations in cortical excitability were shown in patients with epilepsy as well as hyper- or hypoparathyroidism. In patients with primary hyperparathyroidism (pHPT), preoperative elevated serum calcium and parathyroidectomy (PTx) may affect mood and quality of life. We hypothesized that perioperative changes in $\mathrm{Ca}^{2+}$ and PTH in pHPT will affect cortical excitability and improve subjective health.

Design and methods: Transcranial magnetic stimulation (TMS) was performed before and after surgery in 15 pHPT patients. We measured resting motor threshold, cortical silent period (CSP), short intracortical inhibition, and intracortical facilitation. Health questionnaires were administered before, 1 day and 6 months after PTx, along with the disease-specific Pasieka's parathyroid assessment of symptoms (PAS), which was, to our knowledge, its first use in German.

Results: Surgery was successful in all patients. TMS-measurements remained unchanged when analyzing all patients in this pilot study. Postoperatively, depression declined $(P=0.05)$ and quality of life improved significantly $(P=0.001)$ in the SF-36-subscales: vitality, social functioning, mental health and subjective health transition (post-hoc analysis). The PAS proved early relief of disease-specific symptoms $(P<0.001)$.

Conclusions: We found unchanged cortical excitability comparing pre- and post-PTx in this pilot study. Mood and quality of life improved postoperatively. The German PAS is valuable in detecting disease-specific changes early after PTx.
\end{abstract}

\section{Introduction}

In humans, $99 \%$ of the mineral calcium is found in the bones and teeth. The remaining $1 \%$, found in extracellular spaces, has a major influence on the nervous system, and as an intracellular transmitter can modulate ion channel functions throughout the body (1). Calcium metabolism is mainly regulated by parathyroid hormone (PTH), where elevated serum calcium $\left(\mathrm{Ca}^{2+}\right)$ levels result in decreased release of PTH and vice versa.
(C) 2014 European Society of Endocrinology Printed in Great Britain
Intracellular calcium concentration plays a role in the induction and maintenance of acquired epilepsy in status epilepticus (SE), stroke, and traumatic brain injury $(2,3,4)$. These conditions may result in glutamate excitotoxicity and is accompanied by an intracellular increase in calcium concentration levels, resulting in overstimulation of calcium pathways and necrosis $(2,3,5)$. Surviving neurons that are exposed to these altered $\mathrm{Ca}^{2+}$ levels are prone to

Published by Bioscientifica Ltd. 
develop and maintain epileptogenicity $(5,6)$. Correspondingly, voltage-gated calcium channels have been proposed as a target for antiepileptic drugs in preclinical studies (7).

Clinical evidence that altered $\mathrm{Ca}^{2+}$ levels result in changes in cortical excitability has been provided by different case series in hyperparathyroidism (HPT) $(8,9,10)$. Primary HPT ( $\mathrm{pHPT}$ ) consists of continually elevated serum calcium and PTH levels (11), mostly due to a solitary parathyroid adenoma $(1,11,12)$. The only curative treatment for pHPT is the resection of the suspected solitary adenoma(s) or hyperplastic glands (parathyroidectomy (PTx)) to reestablish normocalcemia which may lead to improved quality of life $(13,14)$.

Several case reports show higher amplitudes in somatosensory-evoked potentials (8), electro encephalography (EEG)-alterations, reversible vasoconstriction, and epileptic seizures $(9,15,16)$ or even (nonconvulsive) SE (10) in patients with hypercalcemia. The primary influence of serum calcium, however, remained controversial in earlier papers $(17,18)$. A recent study has delivered repetitive transcranial magnetic stimulation (TMS) and found that both acute and chronic hypercalcemia, as tested respectively in pHPT patients and healthy controls by administering infusions of calcium, modified shortterm synaptic plasticity (19).

On the other hand, cases of epileptic seizures in hypocalcemia have also been reported $(20,21,22,23,24)$ and in one neonatal patient seizures were treated by i.v. calcium administration (25).

Cortical excitability can be accurately measured by TMS. Currently, to our knowledge, only one study has investigated the cortical excitability related to changes in PTX-associated calcium in a predominately conservatively treated group of patients with pHPT (19).

Some studies showed that a successful PTx can have positive effects on different domains of quality of life such as increased energy levels and improved physical as well as emotional well-being $(26,27,28)$. However, other studies found no evidence of a positive change in depression scales when comparing PTx patients with patients who underwent orthopedic surgery (29). Concerning asymptomatic pHPT patients, some randomized control studies found moderate positive effects of operation vs medical care on quality of life $(30,31)$ while others did not (32).

As the role of calcium in cortical excitability is yet to be clarified in humans, pHPT-patients who underwent surgery served as a model for rapid changes in serum calcium in this pilot study. When we investigated the patients, most of them were symptomatic before and after PTx with TMS and administered several mood and quality of life questionnaires up to 6-months' follow-up to identify perioperative changes. Additionally, to our knowledge, this was the first time that a German version of Pasieka's parathyroid assessment of symptoms (PAS) score was administered.

As calcium seems to influence cortical excitability in several ways, we hypothesized that based on the expected transition from hyper- to normocalcemia, postoperative cortical excitability, as measured by TMS, would significantly decrease compared with preoperative measurements. Furthermore, postoperative normocalcemia should lead to an improved quality of life and mood.

\section{Subjects and methods}

\section{Patients}

Twenty right-handed patients (nine males and 11 females) diagnosed with pHPT and scheduled for PTx at the Department of Surgery of the University Hospital Marburg were recruited. They had no history of acute or chronic neurological or psychiatric disease and were not taking medication for CNS indications.

Two female patients withdrew from the study due to TMS intolerance and three patients were excluded because of lack of EMG response, sulcus ulnaris syndrome diagnosis, or postponed parathyroid surgery.

TMS data from the remaining 15 patients (seven males and eight females, median age 55.0 years, range 22-73 years) were obtained before and after PTx. Gender (early: $5 \mathrm{~F}, 6 \mathrm{M}$ vs late: $3 \mathrm{~F}, 2 \mathrm{M}, P=0.742$ ) and age (early: 55.0 (44.0-69.0) vs 59.0 (47.5-70.0), $P=0.462$ ) were equally distributed in the early and late groups. Twelve patients presented with typical symptoms and three were asymptomatic (see Table 1). For the asymptomatic group, the $\mathrm{NIH}$ criteria for operation in asymptomatic pHPT patients were met (33).

TMS measurements after the operation were undertaken more than $30 \mathrm{~h}$ after PTx to adjust to the half time of the typically used narcotics and to eliminate possible effects of anesthesia. Those who were only found to be dosage dependent, as far as we know, were only studied during intraoperative monitoring and not after anesthesia (34).

The study was approved by the Local Ethics Committee of Philipps University of Marburg and written informed consent was obtained from all patients before participation. 
Table 1 Patient characteristics.

\begin{tabular}{|c|c|c|c|}
\hline Patient no. & Age & Gender & $\begin{array}{l}\text { Symptomaticl } \\
\text { asymptomatic }\end{array}$ \\
\hline 3 & 22 & $\mathrm{M}$ & Symptomatic \\
\hline 4 & 46 & $\mathrm{M}$ & Symptomatic \\
\hline 7 & 72 & $\mathrm{M}$ & Symptomatic \\
\hline 8 & 55 & $\mathrm{~F}$ & Symptomatic \\
\hline 9 & 66 & $\mathrm{M}$ & Symptomatic \\
\hline 15 & 59 & $F$ & Symptomatic \\
\hline 17 & 40 & $\mathrm{~F}$ & Symptomatic \\
\hline 23 & 44 & $\mathrm{~F}$ & Symptomatic \\
\hline 27 & 58 & $\mathrm{M}$ & Symptomatic \\
\hline 33 & 69 & $F$ & Symptomatic \\
\hline 54 & 49 & $\mathrm{M}$ & Asymptomatic \\
\hline 60 & 73 & $F$ & Symptomatic \\
\hline 69 & 71 & $\mathrm{~F}$ & Asymptomatic \\
\hline 70 & 37 & $\mathrm{~F}$ & Asymptomatic \\
\hline 84 & 52 & $\mathrm{M}$ & Symptomatic \\
\hline
\end{tabular}

Symptoms

Recurring renal colic

Kidney stones, renal colic

Renal colic

Stomach trouble, osteoporosis

Acute pancreatitis, $\mathrm{Ca}>0.2 \mathrm{mmol}$ above norm

Bone pain, renal colic

Depression, explicit operation wish

Bone pain, tiredness, depression

Pancreatitis

Bone pain

Bone pain

Fulfills NIH criteria ${ }^{a}$ for asymptomatic pHPT with

$\mathrm{Ca}>0.2 \mathrm{mmol}$ above norm

Fulfills NIH criteria ${ }^{a}$ for asymptomatic pHPT with $\mathrm{Ca}>0.2 \mathrm{mmol}$ above norm

Fatigue/depression, polyuria

${ }^{a}$ As defined in the article of Bilezikian et al. (33).

\section{Transcranial magnetic stimulation}

TMS was administered on the day before surgery ( $-1 \pm 0$ days) as well as after a median of 3 days ( 1 day, 25 th quartile-142 days, 75 th quartile) for all subjects. Subjects were comfortably seated in an armchair with their head fixed in a custom plastic foam headrest. TMS was delivered through a focal figure-of-eight-shaped magnetic coil (70 mm external loop diameter) connected to two Magstim 200 magnetic stimulators via a BiStim-module (all Magstim, Whitland, Dyfed, UK). The coil was placed flat on the head over the left motor cortex, approximately at an angle of $45^{\circ}$ to the sagittal plane, inducing a current in the brain region roughly perpendicular to the central sulcus, flowing from posterior to anterior, as this has been reported to be the most effective way to activate the corticospinal system transsynaptically (35). Motor-evoked potentials (MEP) were recorded using surface EMG Ag/ $\mathrm{AgCl}$ electrodes placed over the right abductor digiti minimi muscle using a belly-tendon montage. The raw signal was amplified, filtered (10-20 kHz), and recorded with a PC using a commercially available data-collection and averaging program (Magnetix, Centre of Sensorimotor Research, Munich, Germany) for offline analysis. The optimal coil placement was determined by recording MEP while varying the coil position. The coil position leading to the highest peak-to-peak amplitude of the MEP ('hot spot') was marked with a semi-permanent pen directly on the scalp to ensure accurate coil positioning during testing.

All sessions followed a fixed sequence of TMS measurements: first, the test stimulus (TS) and resting motor threshold (RMT), and then the paired-pulse parameters, short intracortical inhibition (SICI) and intracortical facilitation (ICF), were obtained in a random order. In all paired-pulse TMS procedures, the interval between trials was randomly changed between 4 and $6 \mathrm{~s}$; in single-pulse procedures the inter-trial interval was $5 \mathrm{~s}$. The protocol was concluded with determination of the cortical silent period (CSP).

While RMT and ICF are excitatory parameters, SICI and CSP determine the inhibitory phenomena. All of those show changes at different neurotransmitter systems (36). Voltage-gated sodium channel mechanisms influence the RMT. SICI and ICF are influenced by glutamatergic and the $\mathrm{GABA}_{\mathrm{A}}$ systems in the cortex. The CSP shows inhibitory $\mathrm{GABA}_{\mathrm{B}}$-mediated long-term mechanisms, and these are probably also responsible for late intracortical inhibition (LICI).

\section{TMS parameters}

TMS parameters were specified as follows:

i) The RMT was defined as the lowest stimulator output intensity required to induce MEP peak-to-peak amplitude $>50 \mu \mathrm{V}$ in at least five of ten consecutive trials. Complete muscle relaxation was monitored via audiovisual feedback. A step-by-step intensity resolution of the maximal stimulator output was used for the determination of the individual RMT using the maximum likelihood threshold hunting (MLTH) procedure for TMS (@ Dr Friedemann Awiszus, Magdeburg (37)). 
ii) SICI and ICF were obtained with paired-pulse TMS. A conditioning and a TS were applied with different fixed interstimulus intervals (ISI). The conditioning stimulus was set to an intensity of $75 \%$ of the RMT, as this does not produce changes in excitability in the spinal cord $(38,39)$. The intensity of the following suprathreshold TS was adjusted to produce MEPs of $\sim 1.5 \mathrm{mV}$ peak-to-peak amplitude if delivered without a preceding conditioning stimulus (TS). SICI was obtained at short ISIs of 2 and $3 \mathrm{~ms}$, leading to a decreased MEP compared with an MEP induced by a nonconditioned TS. ICF was obtained using ISIs of 10 and $15 \mathrm{~ms}$, leading to an increased MEP $(35,36,39,40,41)$. Owing to good correlation of the inhibitory ISIs of 2 and $3 \mathrm{~ms}$ (pre: $\rho=0.696, P=0.004$ and post: $\rho=0.892, P<0.001)$, we computed one variable (ICI) and did the same for ISIs of 10 and $15 \mathrm{~ms}$ (pre: $\rho=0.948, P<0.001$ and post: $\rho=0.876, P<0.001$ ), which are measures of ICF. LICI was tested at ISI of $150 \mathrm{~ms}$ and two equal stimuli of $150 \%$ RMT were delivered. Fifteen trials of single nonconditioned test stimuli and 15 paired stimuli of each ISI, generated in random order by the computer software, were recorded. The average of the 15 trials was used to define the amplitude of the peakto-peak MEP for each condition. The conditioned response was defined as the mean amplitude of the conditioned responses belonging to each ISI, expressed as the percentage of the mean amplitude of the unconditioned test response. The CSP was measured during 20 trials at a stimulus intensity of $110 \%$ of the RMT. Participants were instructed to hold a voluntary muscle contraction of $\sim 30 \%$ of their maximal force, controlled by audio-visual feedback. CSP duration was determined offline in two ways. For visually guided analysis, the CSP duration was defined as the time from TMS stimulus artefact to the first reoccurrence of voluntary EMG activity exceeding $25 \%$ of muscle activity before the stimulus. This was always determined by the same investigator to minimize variability. Duration was determined offline in the Magnetix program.

One value per person and session was calculated by averaging separately for RMT, SICI, ICF, LICI, and CSP.

\section{Emotional well-being, quality of life}

Questionnaires on subjective health and mood status were filled in by the patients on the day before operation, 1 day after operation and half a year after operation. The Beck Depression Inventory (BDI-II) (42) measures levels of depression on a four-point Likert scale for 21 statements. The patient is asked to mark the statement that best fits his mood for the past 2 weeks ( $0-3$ points). The sum of scores determines the degree of depression, with a score equal to or above 14 being clinically relevant (maximum 63 points). The Short Form 36 Health Survey (SF-36 $(43,44)$ ) is an instrument used to determine individual nondiseasespecific health in patients. At the level of raw scores, higher scores indicate more impairment except for selfreported health transition, whereas higher values indicate negative health development in the last year. Several subscores and two components, mental (mental component score (MCS)) and physical health (physical component score (PCS)) (45), can be measured.

Pasieka's PAS score (46) was applied to detect specific symptoms in the pHPT patient group. The PAS measures 13 aspects of parathyroidism that are disease-specific (e.g. bone pain, mood alteration, degree of weakness, and being forgetful) on a visual analog scale (47) with a maximum score of 1300. Lower scores refer to less disturbing symptoms. Additionally, two scores on quality of life and well-being can be scored separately (maximum each ten points). As no German version of the PAS was readily available, items were translated by a native English speaker.

\section{Clinical parameters}

The serum calcium and PTH levels, as well as clinical data including medication and symptoms of hyper- and hypocalcemia, were determined on the day of admission to the ward and following PTx. Grip force was measured after each TMS session. Each patient squeezed a hydraulic hand dynamometer (Saehan Corp., Masan, Korea) at maximum power with their dominant hand.

The F-waves $(n=5)$ and nerve conduction velocity $(n=7)$ were measured to discover possible peripheral nerve lesions or polyneuropathy and reduce confounding with the results of TMS measurements.

\section{Statistical analysis}

The analysis was conducted using PASW Statistics 18-20 (SPSS, IBM Company). Data are expressed as median \pm 25 th and 75 th percentiles or s.D. Owing to sample size and skewed data, we chose to use nonparametric statistical tests. The Mann-Whitney $U$ test was applied to independent samples and the Wilcoxon's rank sum test for paired samples. To determine associations between TMS 
Table 2 TMS parameters for the entire group $(n=15)$, pre- and post-PTx as measured by Wilcoxon's test.

\begin{tabular}{|c|c|c|c|}
\hline & Pre-PTx & Post-PTx & $\boldsymbol{P}$ \\
\hline RMT & $44.0(42.0-51.0)$ & $45.0(38.0-54.0)$ & 0.711 \\
\hline $\mathrm{ICF}$ & $135.8(113.3-220.1)$ & 137.5 (99.5-174.6) & 0.910 \\
\hline $\mathrm{SICl}$ & 46.8 (35.5-94.7) & 49.4 (37.8-70.0) & 0.910 \\
\hline $\mathrm{LICl}$ & $73.1(52.2-89.0)$ & 76.7 (66.2-88.8) & 0.394 \\
\hline CSP & $94.9(74.3-124.4)$ & $100.6(80.6-113.1)$ & 0.552 \\
\hline
\end{tabular}

PTx, parathyroidectomy; RMT, resting motor threshold (in \% of maximal stimulator output); ICF, intracortical facilitation; SICl, short intracortical inhibition; LICl, late intracortical inhibition; CSP, cortical silent period (ms); ICF, ICl, and $\mathrm{LICl}$ all as \% of unconditioned MEP; numbers are displayed as median (25th-75th quartiles).

parameters and $\mathrm{Ca}^{2+}$ and PTH, Spearman's correlation coefficient $(\rho)$ was used.

Multivariate ANOVA (MANOVA) was applied with the three-staged within-subject factor time (pre, post, and 6-months' follow-up) and questionnaire-specific dependent variables. If group variances were nonhomogenous (according to Mauchly's test of sphericity), degrees of freedom were adjusted (Greenhouse-Geisser). When appropriate, either univariate ANOVA or post-hoc tests (Bonferroni's adjusted) are reported.

Not all participants returned all questionnaires. This resulted in reduced data (BDI, $n=12$ and SF-36, $n=13$ ). For determination of PAS changes, we left out one patient due to outlier responses $(n=12)$.

\section{Results}

\section{Hormone levels and clinical variables}

A significant reduction in $\mathrm{Ca}^{2+}$ and PTH level was achieved by surgery from pre- to post-measurement $\left(\mathrm{Ca}^{2+}\right.$ pre: 2.8 (2.6-2.9) vs post: 2.3 (2.2-2.5), $P=0.004$; PTH pre: 133.00 (107.00-170.00) vs post: 12.00 (6.75-23.5), $P=0.002)$. Postoperatively, $\mathrm{Ca}^{2+}$ and PTH were significantly correlated $(r=-0.704, P=0.011)$. Grip force (33.50 (27.5-43.75) vs $36.00(27.50-44.00), P=0.721)$ as well as F-waves $(P=0.236)$ and nerve conduction velocity $(P=0.144)$ remained stable from pre- to post-operation.

\section{Cortical excitability}

We found significant correlations between the two measurements for the following TMS parameters: TS $(\rho=0.810, P<0.001)$, RMT $(\rho=0.649, P=0.009)$, SICI $(\rho=0.607, P=0.016)$, and ICF $(\rho=0.504, P=056)$.
$\mathrm{Ca}^{2+}$ and PTH, however, showed no consistent correlations with any TMS parameters, either pre- or post-PTx.

There were no significant alterations in the TMS parameters of the entire group when comparing pre- and post-PTx (see Table 2).

\section{Emotion and well-being}

Multivariate repeated measure analysis (RM-MANOVA) with the BDI score as the dependent variable and time as the within-subject-factor indicated an overall decline in depressive symptoms over time ( $P=0.05$, see Table 3$)$. Univariate analysis revealed that these symptoms remained stable from pre- to immediately post-PTx $(P=0.192)$ but that patients were significantly less depressed at 6-months' follow-up compared with pre- and post-measurements ( $P=0.018$ and $P=0.039$ respectively).

The analysis of the overall effects of all subscales in the SF-36 revealed a multivariate effect for 'time' $(F(18,28)=$ $\left.3.65, \eta^{2}=0.701, P=0.001\right)$. We subsequently determined the significantly altered subscores in univariate analysis. These showed significant improvements in vitality, social functioning, mental health, and subjective health transition $(P<0.001, P=0.016, P=0.042$, and $P=0.001$ respectively, see Fig. 1). Post-hoc analysis clarified that with the exception of mental health, which only changed from post-PTx to 6-months' follow-up, all the other scores improved significantly from pre- to post-PTx and from pre-PTx to 6-months' follow-up, while no significant immediate-pre-post changes were found (see Fig. 1). When separately analyzing the subscales of the PCS and MCS, we found no significant changes across measurements.

\section{Disease-specific PAS score}

Analysis revealed a decline in PAS symptoms over time $(n=12, P<0.001)$. However, univariate analysis showed a decline only from pre- to immediately post-PTx (pre-PTx,

Table 3 Change in BDI and PAS over time.

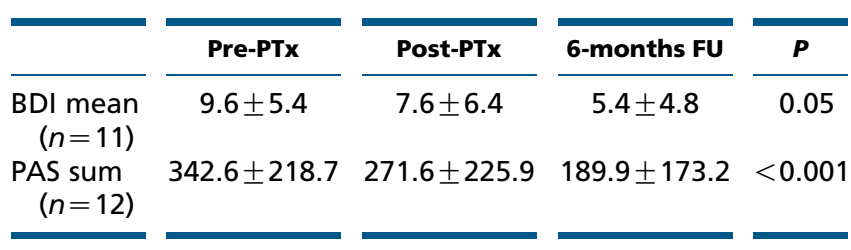

BDI, Beck Depression Inventory; PAS, parathyroidect assessment of symptoms scores; pre, 1 day before parathyroidectomy (PTx); post, 1 day after PTx; FU, follow-up; data are displayed as median \pm s.D., multivariate $P$ is given. 


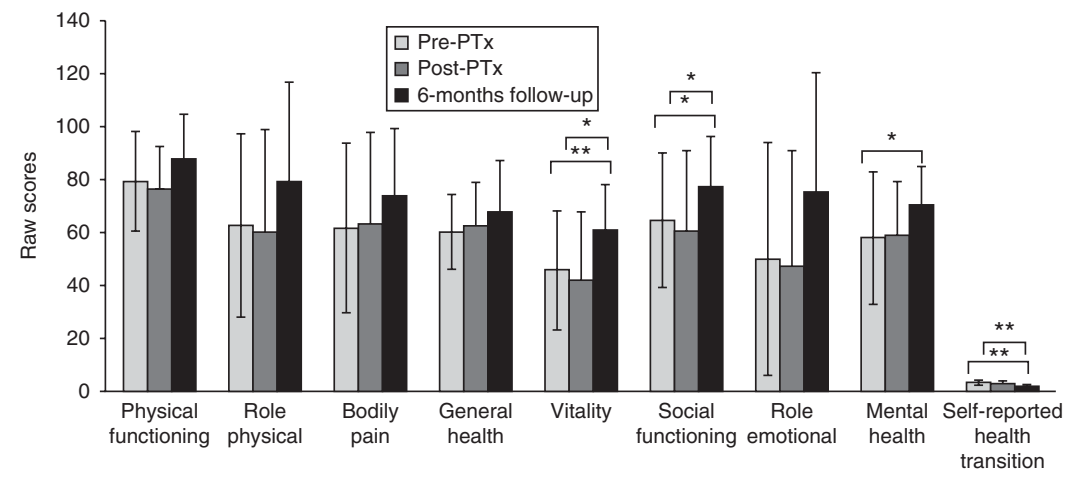

\section{Figure 1}

Subscales of SF-36 for pre, post, and 6-months (FU; $n=12$ ). In post-hoc analysis vitality, social functioning, and selfreported health transition differed from pre to FU and post to FU. Mental health changed from post to FU only. Note that

$342.58 \pm 218.69$; post-PTx, 271.58 $\pm 225.90, P=0.047)$. The PAS furthermore showed strong overall correlations between the immediate post-measurement and the 6-months' follow-up $(\rho=0.983, P<0.001)$, indicating that long-term postoperative improvement can be predicted by the early postoperative PAS score. The two PAS subscores for quality of life and well-being were also highly correlated with both measurements after the operation ( $\rho=0.849, P=016$ and $\rho=0.934, P=0.002$ respectively).

All questionnaires correlated well in the vast majority of domains at the three times of measurement. This included amongst others very good correlations of the BDI and PAS on all inquiries (pre $\rho=0.778, P=0.002$; post $\rho=0.920, P=0.000$; and 6 months $\rho=0.862, P=0.000$ ).

Our patient group displayed good to strong correlations between PAS and SF-36 for the following subscales: general health, vitality, social functioning, role emotional, and mental health (see Table 4) but none with all eight subscales and the overall change in health.

We found that calcium correlated after the operation with a decrease in depressive symptoms on BDI $(r=-0.633, P=0.027)$ and an improvement in PAS wellbeing $(r=0.809, P=0.005)$. Several SF-36 scales (role physical, overall health, vitality, emotional well-being) correlated with postoperative calcium $(r=0.670, P=0.009$; $r=0.619, P=0.024 ; r=0.712, P=0.004 ;$ and $r=0.731$, $P=0.003$ respectively). There were no correlations of calcium and any BDI or PAS-score before the operation. For SF-36, we found preoperative correlations with calcium on the subscale physical functioning. higher scores mean more impairment except for self-reported health transition, where fewer points are positive. Median and S.D. are displayed. PTx, parathyroidectomy, ${ }^{*} P<0.05$ and $\star * P<0.001$.

\section{Discussion}

This study collected data on perioperative changes in cortical excitability measured by TMS and changes in mood and quality of life following successful PTx and consecutive normalization of serum calcium levels from patients with pHPT. We aimed to understand calciumdependent alterations by using pHPT as a model for rapid calcium changes in humans. Therefore, cortical

Table 4 Significant correlations between PAS and SF-36 subscales.

\begin{tabular}{|c|c|c|c|}
\hline & \multicolumn{3}{|c|}{ PAS } \\
\hline & Pre & Post & 6 months \\
\hline \multicolumn{4}{|l|}{ SF-36 pre } \\
\hline General health & $0.565^{*}$ & & \\
\hline Vitality & $0.754^{*}$ & & \\
\hline Social functioning & $0.742 *$ & & \\
\hline Role emotional & $0.749 *$ & & \\
\hline Mental health & $0.844^{\dagger}$ & & \\
\hline \multicolumn{4}{|l|}{ SF-36 post } \\
\hline General health & & $0.711 *$ & \\
\hline Vitality & & $0.774 *$ & \\
\hline Social functioning & & $0.685^{*}$ & \\
\hline Role emotional & & $0.682 *$ & \\
\hline Mental health & & $0.757^{*}$ & \\
\hline \multicolumn{4}{|l|}{ SF-36 6 months } \\
\hline General health & & & $0.887^{\dagger}$ \\
\hline Vitality & & & $0.931^{\dagger}$ \\
\hline Social functioning & & & $0.670 *$ \\
\hline Role emotional & & & $0.758^{*}$ \\
\hline Mental health & & & $0.910^{\dagger}$ \\
\hline
\end{tabular}


excitability was measured pre- and postoperatively in each patient. Changes in mood and quality of life following PTx up to 6-months' follow-up were investigated. This is, to our knowledge, the first study to use a German version of the disease-specific Pasieka's PAS score.

TMS measurement was performed in all patients before PTx to obtain data during a hypercalcemic state and after PTx to collect data on cortical excitability during normocalcemia. Using this within-subject design restricted confounding interindividual factors that arise when comparing different patients. Surgery was successful and all 15 patients who completed the study showed a significant reduction in both $\mathrm{Ca}^{2+}$ and PTH levels into normal range postoperatively.

Cortical excitability showed strong correlations among TMS parameters for both sessions, affirming the relatively stable intra-individual cortical excitability. Considering the whole group of patients, we did not find significant alterations in cortical excitability as measured by TMS. At least for hypercalcemia, it has been reported that EEG patterns normalize gradually after treatment with hypocalcemic agents (4). Lacovelli et al. (19) in their rTMS study reported only two pHPT patients undergoing PTx that showed unchanged RMT, CSP, and MEP amplitude but improved MEP facilitation after PTx. We could not replicate this finding in a larger cohort.

Changes in patients' overall health as well as mental health were positive after PTx. Promisingly, the PAS, designed as a disease-specific tool, showed a decline from pre- to immediately post-PTx and strong correlations between immediate post-operative measurement and a longer 6-months' follow-up. This therefore underlines the rapid positive impact of the surgical intervention (28) even though, due to our study design, we can't compare with a surgical control-group. The PAS therefore seems an excellent tool during the hospital phase for early prediction of changes in specific symptoms. Ongoing postsurgical recovery or experience of surgery on the first postoperative day might have influenced the unchanged depression scores between pre- and immediately postoperation. When, in a different study, first measurement was held at two weeks, postoperatively depressive symptoms had declined (48). Similarly, our longer follow-up showed a decline from both earlier measurements to 6-months' follow-up and therefore we conclude that depressive symptoms normalize over time. Depressed mood is one of the clinically relevant neuropsychiatric symptoms in pHPT, while the influence of calcium on depressive symptoms remains unclear $(29,49)$. Like Chiang et al. (29), we found a slight and diverse evidence of any dependency of the questionnaires and cortical excitability on calcium levels, while recent research has found a correlation of $\mathrm{CA}^{2+}$ and depression, suicidal tendencies and quality of life (48). This might stem from the larger multicenter cohort of Weber et al. and should be verified in future studies.

Similar to Mihai \& Sadler (27), who showed a good correlation between the well-established SF-36 and the original English PAS, we were able to show a good correlation for both German versions. Our data emphasize that its administration before and immediately after PTx yields reliable insights into specific changes and that longterm follow-up might not be required as the data did not subsequently change. This should be confirmed by prospective trials with a larger number of patients. The disease-specific items and the faster administration time of the latter should promote the use of PAS in surgical settings for quick and objective assessment of perioperative changes in pHPT.

Limitations of our study are the lack of any control group to better distinguish the results as the effects of PTx, unspecific surgery, or disease relief effects. Furthermore, the number of enrolled patients is rather small, which led to slightly underpowered TMS measurements.

Further research should include a larger number of patients with pHPT. Additionally, cortical excitability associated with hypocalcemia should be measured as hypocalcemia, or a rapid change in calcium balance after prolonged hypercalcemia (50) in certain cases, may correlate with a decrease in seizure threshold and is consistent with studies on both rodent hippocampal slices (51) and human case reports $(11,51,52)$. This might influence the clinical treatment of an altered calcium balance. A control group should be included, preferably both a nonsurgical as well as surgical group, to differentiate the effects of disease cure, anesthesia, and surgery especially in the mood domain.

Declaration of interest

The authors declare that there is no conflict of interest that could be perceived as prejudicing the impartiality of the research reported.

\section{Funding}

This research did not receive any specific grant from any funding agency in the public, commercial or not-for-profit sector.

\section{Acknowledgements}

The authors thank B Norwood for helpful comments. 


\section{References}

1 Lang F \& Murer H. Kalzium-und Phosphathaushalt. In Physiologie des Menschen, pp 740-752. Eds R Schmidt, F Lang \& G Thews. Heidelberg: Springer Verlag, 2005.

2 DeLorenzo RJ \& Sun DA. Basic mechanisms in status epilepticus: role of calcium in neuronal injury and the induction of epileptogenesis. Advances in Neurology 200697 187-197.

3 Raza M, Blair RE, Sombati S, Carter DS, Deshpande LS \& DeLorenzo RJ. Evidence that injury-induced changes in hippocampal neuronal calcium dynamics during epileptogenesis cause acquired epilepsy. PNAS 2004101 17522-17527. (doi:10.1073/pnas.0408155101)

4 Castilla-Guerra L, del Carmen Fernandez-Moreno M, Lopez-Chozas JM \& Fernandez-Bolanos R. Electrolytes disturbances and seizures. Epilepsia 200647 1990-1998. (doi:10.1111/j.1528-1167.2006.00861.x)

5 DeLorenzo RJ, Sun DA \& Deshpande LS. Erratum to “Cellular mechanisms underlying acquired epilepsy: the calcium hypothesis of the induction and maintenance of epilepsy" (Pharmacol. Ther. 105(3) (2005) 229-266). Pharmacology \& Therapeutics 2006111 288-325. (doi:10.1016/j.pharmthera.2004.10.015)

6 Kulak W, Sobaniec W, Wojtal K \& Czuczwar SJ. Calcium modulation in epilepsy. Polish Journal of Pharmacology 200456 29-41. (doi:10.1211/ 002235704777489302)

7 Stefani A, Spadoni F \& Bernardi G. Voltage-activated calcium channels: targets of antiepileptic drug therapy? Epilepsia 199738 959-965. (doi:10.1111/j.1528-1157.1997.tb01477.x)

8 Kanda F, Jinnai J \& Fujita T. Somatosensory evoked potentials in patients with hypocalcaemia after parathyroidectomy. Journal of Neurology 1988235 136-139. (doi:10.1007/BF00314302)

9 Cherry TA, Kauffman RP \& Myles TD. Primary hyperparathyroidism, hypercalcemic crisis and subsequent seizures occurring during pregnancy: a case report. Journal of Maternal-fetal \& Neonatal Medicine 200212 349-352. (doi:10.1080/jmf.12.5.349.352)

10 Kumpfel T, Lechner C, Auer D, Kraft E, Lydtin H \& Trenkwalder C. Non-convulsive status epilepticus with marked neuropsychiatric manifestations and MRI changes after treatment of hypercalcaemia. Acta Neurologica Scandinavica 2000102 337-339. (doi:10.1034/j.16000404.2000.102005337.x)

11 Deftos LJ, Parthemore JG \& Stabile BE. Management of primary hyperparathyroidism. Annual Review of Medicine 199344 19-26. (doi:10.1146/annurev.me.44.020193.000315)

12 Fraser WD. Hyperparathyroidism. Lancet 2009374 145-158. (doi:10.1016/S0140-6736(09)60507-9)

13 Karakas E, Steinfeldt T, Gockel A, Westermann R, Kiefer A \& Bartsch DK. Transoral thyroid and parathyroid surgery. Surgical Endoscopy 201024 1261-1267. (doi:10.1007/s00464-009-0757-z)

14 Hasse C, Sitter H, Brune M, Wollenteit I, Nies C \& Rothmund M. Quality of life and patient satisfaction after reoperation for primary hyperparathyroidism: analysis of long-term results. World Journal of Surgery 200226 1029-1036. (doi:10.1007/s00268-002-6664-2)

15 Thiel R. Might calcium disorders cause or contribute to myoclonic seizures in epileptics? Medical Hypotheses $2006 \mathbf{6 6} 969-974$. (doi:10.1016/j.mehy.2005.11.018)

16 Chen TH, Huang CC, Chang YY, Chen YF, Chen WH \& Lai SL. Vasoconstriction as the etiology of hypercalcemia-induced seizures. Epilepsia 200445 551-554. (doi:10.1111/j.0013-9580.2004.57003.x)

17 Cohn R \& Sode J. The EEG in hypercalcemia. Neurology 197121 154-161. (doi:10.1212/WNL.21.2.154)

18 Goldstein DA, Feinstein EI, Chui LA, Pattabhiraman R \& Massry SG. The relationship between the abnormalities in electroencephalogram and blood levels of parathyroid hormone in dialysis patients. Journal of Clinical Endocrinology and Metabolism 198051 130-134. (doi:10.1210/ jcem-51-1-130)

19 Iacovelli E, Gilio F, Mascia ML, Scillitani A, Romagnoli E, Pichiorri F, Fucile S, Minisola S \& Inghilleri M. Acute and chronic effects of hypercalcaemia on cortical excitability as studied by $5 \mathrm{~Hz}$ repetitive transcranial magnetic stimulation. Journal of Physiology 2011589 1619-1626. (doi:10.1113/jphysiol.2010.201111)

20 Kline CA, Esekogwu VI, Henderson SO \& Newton KI. Non-convulsive status epilepticus in a patient with hypocalcemia. Journal of Emergency Medicine 199816 715-718. (doi:10.1016/S0736-4679(98)00089-4)

21 Fonseca OA \& Calverley JR. Neurological manifestations of hypoparathyroidism. Archives of Internal Medicine 1967120 202-206. (doi:10.1001/archinte.1967.00300020074009)

22 Dimich A, Bedrossian PB \& Wallach S. Hypoparathyroidism. Clinical observations in 34 patients. Archives of Internal Medicine 1967120 449-458. (doi:10.1001/archinte.1967.04410010063009)

23 Bindu M \& Harinarayana CV. Hypoparathyroidism: a rare treatable cause of epilepsy - report of two cases. European Journal of Neurology 200613 786-788. (doi:10.1111/j.1468-1331.2006.01287.x)

24 Rosenow F \& Reis J. Schilddrüse, Nebenschilddrüse und Epilepsie. Zeitschrift für Epileptologie 200619 183-186. (doi:10.1007/ s10309-006-0208-y)

25 Kossoff EH, Silvia MT, Maret A, Carakushansky M \& Vining EP. Neonatal hypocalcemic seizures: case report and literature review. Journal of Child Neurology 200217 236-239. (doi:10.1177/ 088307380201700319)

26 Coker LH, Rorie K, Cantley L, Kirkland K, Stump D, Burbank N, Tembreull T, Williamson J \& Perrier N. Primary hyperparathyroidism, cognition, and health-related quality of life. Annals of Surgery 2005242 642-650. (doi:10.1097/01.sla.0000186337.83407.ec)

27 Mihai R \& Sadler GP. Pasieka's parathyroid symptoms scores correlate with SF-36 scores in patients undergoing surgery for primary hyperparathyroidism. World Journal of Surgery 200832 807-814. (doi:10.1007/s00268-008-9509-9)

28 Pasieka JL \& Parsons LL. Prospective surgical outcome study of relief of symptoms following surgery in patients with primary hyperparathyroidism. World Journal of Surgery 199822 513-518 (discussion 518-519). (doi:10.1007/s002689900428)

29 Chiang CY, Andrewes DG, Anderson D, Devere M, Schweitzer I \& Zajac JD. A controlled, prospective study of neuropsychological outcomes post parathyroidectomy in primary hyperparathyroid patients. Clinical Endocrinology 200562 99-104. (doi:10.1111/ j.1365-2265.2004.02180.x)

30 Ambrogini E, Cetani F, Cianferotti L, Vignali E, Banti C, Viccica G, Oppo A, Miccoli P, Berti P, Bilezikian JP et al. Surgery or surveillance for mild asymptomatic primary hyperparathyroidism: a prospective, randomized clinical trial. Journal of Clinical Endocrinology and Metabolism 200792 3114-3121. (doi:10.1210/jc.2007-0219)

31 Rao DS, Phillips ER, Divine GW \& Talpos GB. Randomized controlled clinical trial of surgery versus no surgery in patients with mild asymptomatic primary hyperparathyroidism. Journal of Clinical Endocrinology and Metabolism 200489 5415-5422. (doi:10.1210/ jc.2004-0028)

32 Bollerslev J, Jansson S, Mollerup CL, Nordenstrom J, Lundgren E, Torring O, Varhaug JE, Baranowski M, Aanderud S, Franco C et al. Medical observation, compared with parathyroidectomy, for asymptomatic primary hyperparathyroidism: a prospective, randomized trial. Journal of Clinical Endocrinology and Metabolism 200792 1687-1692. (doi:10.1210/jc.2006-1836)

33 Bilezikian JP, Khan AA \& Potts JT Jr. Guidelines for the management of asymptomatic primary hyperparathyroidism: summary statement from the third international workshop. Journal of Clinical Endocrinology and Metabolism 200994 335-339. (doi:10.1210/jc.2008-1763)

34 Ziemann U. TMS and drugs. Clinical Neurophysiology 2004115 1717-1729. (doi:10.1016/j.clinph.2004.03.006)

35 Brasil-Neto JP, McShane LM, Fuhr P, Hallett M \& Cohen LG. Topographic mapping of the human motor cortex with magnetic stimulation: factors affecting accuracy and reproducibility. Electroencephalography and Clinical Neurophysiology 1992 85 9-16. (doi:10.1016/0168-5597(92)90095-S) 
36 Nakamura H, Kitagawa H, Kawaguchi Y \& Tsuji H. Intracortical facilitation and inhibition after transcranial magnetic stimulation in conscious humans. Journal of Physiology 1997498 817-823.

37 Awiszus F. TMS and threshold hunting. Supplements to Clinical Neurophysiology 200356 13-23.

38 Di Lazzaro V, Restuccia D, Oliviero A, Profice P, Ferrara L, Insola A, Mazzone P, Tonali P \& Rothwell JC. Magnetic transcranial stimulation at intensities below active motor threshold activates intracortical inhibitory circuits. Experimental Brain Research 1998119 265-268. (doi:10.1007/s002210050341)

39 Kujirai T, Caramia MD, Rothwell JC, Day BL, Thompson PD, Ferbert A, Wroe S, Asselman P \& Marsden CD. Corticocortical inhibition in human motor cortex. Journal of Physiology $1993 \mathbf{4 7 1}$ 501-519.

40 Reis J, Wentrup A, Hamer HM, Mueller HH, Knake S, Tergau F, Oertel WH \& Rosenow F. Levetiracetam influences human motor cortex excitability mainly by modulation of ion channel function a TMS study. Epilepsy Research 200462 41-51. (doi:10.1016/ j.eplepsyres.2004.08.001)

41 Werhahn KJ, Kunesch E, Noachtar S, Benecke R \& Classen J. Differential effects on motorcortical inhibition induced by blockade of GABA uptake in humans. Journal of Physiology 1999517 591-597. (doi:10.1111/j.1469-7793.1999.0591t.x)

42 Beck AT, Steer RA \& Brown GK. In Manual for the Beck Depression Inventory-II. San Antonio, TX: Psychological Corporation, 1996.

43 SAGE Publications I. SF-36 and SF-12 Health Surveys. Encyclopedia of Medical Decision Making. SAGE Publications, Inc. Thousand Oaks, CA: SAGE Publications, Inc.

44 Ware JE Jr, Kosinski M, Bayliss MS, McHorney CA, Rogers WH \& Raczek A. Comparison of methods for the scoring and statistical analysis of SF-36 health profile and summary measures: summary of results from the Medical Outcomes Study. Medical Care 199533 AS264-AS279. (doi:10.1097/00005650-199501001-00005)
45 Ware JE \& Kosinski M. Interpreting SF-36 summary health measures: a response. Quality of Life Research 200110 405-413 (discussion 415-420). (doi:10.1023/A:1012588218728)

46 Pasieka JL, Parsons LL, Demeure MJ, Wilson S, Malycha P, Jones J \& Krzywda B. Patient-based surgical outcome tool demonstrating alleviation of symptoms following parathyroidectomy in patients with primary hyperparathyroidism. World Journal of Surgery 200226 942-949. (doi:10.1007/s00268-002-6623-y)

47 Caron NR \& Pasieka JL. What symptom improvement can be expected after operation for primary hyperparathyroidism? World Journal of Surgery 200933 2244-2255. (doi:10.1007/s00268-009-9987-4)

48 Weber T, Eberle J, Messelhauser U, Schiffmann L, Nies C, Schabram J, Zielke A, Holzer K, Rottler E, Henne-Bruns D et al. Parathyroidectomy, elevated depression scores, and suicidal ideation in patients with primary hyperparathyroidism: results of a prospective multicenter study. JAMA Surgery 2013148 109-115. (doi:10.1001/2013. jamasurg.316)

49 Walker MD, McMahon DJ, Inabnet WB, Lazar RM, Brown I, Vardy S, Cosman F \& Silverberg SJ. Neuropsychological features in primary hyperparathyroidism: a prospective study. Journal of Clinical Endocrinology and Metabolism 200994 1951-1958. (doi:10.1210/jc.2008-2574)

50 Fonseca VA, Bloom RD, Dick R \& Dandona P. Tetany despite normocalcaemia and normomagnesaemia following parathyroidectomy. Postgraduate Medical Journal 198763 885-886. (doi:10.1136/ pgmj.63.744.885)

51 Wang T, Wang J, Cottrell JE \& Kass IS. Small physiologic changes in calcium and magnesium alter excitability and burst firing of CA1 pyramidal cells in rat hippocampal slices. Journal of Neurosurgical Anesthesiology 200416 201-209. (doi:10.1097/00008506200407000-00004)

52 Mrowka M, Knake S, Klinge H, Odin P \& Rosenow F. Hypocalcemic generalised seizures as a manifestation of iatrogenic hypoparathyroidism months to years after thyroid surgery. Epileptic Disorders: International Epilepsy Journal with Videotape 20046 85-87.

Received 2 July 2013

Revised version received 27 September 2013

Accepted 30 October 2013 